\title{
Probing Charges on the Atomic Scale by Means of Atomic Force Microscopy
}

\author{
F. Albrecht and J. Repp* \\ Institute of Experimental and Applied Physics, University of Regensburg, 93053 Regensburg, Germany \\ M. Fleischmann and M. Scheer \\ Institute of Inorganic Chemistry, University of Regensburg, 93053 Regensburg, Germany \\ M. Ondráček and P. Jelínek \\ Institute of Physics of the Academy of Sciences of the Czech Republic, 16253 Prague, Czech Republic
}

(Received 27 February 2015; revised manuscript received 1 July 2015; published 13 August 2015)

\begin{abstract}
Kelvin probe force spectroscopy was used to characterize the charge distribution of individual molecules with polar bonds. Whereas this technique represents the charge distribution with moderate resolution for large tip-molecule separations, it fails for short distances. Here, we introduce a novel local force spectroscopy technique which allows one to better disentangle electrostatic from other contributions in the force signal. It enables one to obtain charge-related maps at even closer tip-sample distances, where the lateral resolution is further enhanced. This enhanced resolution allows one to resolve contrast variations along individual polar bonds.
\end{abstract}

DOI: 10.1103/PhysRevLett.115.076101

PACS numbers: 68.37.-d, 68.43.-h

The use of a well-defined functionalization of a scanning probe tip with one $\mathrm{CO}$ molecule has enabled submolecular resolution in frequency-modulated atomic force microscopy (FM-AFM) resolving the chemical structure of individual molecules [1]. Since then, this technique has been widely applied [2-10]. AFM-based Kelvin probe force spectroscopy (KPFS) [11] provides the local contact potential difference (LCPD) between tip and sample [12-14]. As surface charges and dipoles affect the local work function, LCPD is intimately linked to the charge distribution at surfaces $[15,16]$ such that KPFS can be used to determine the charge state of individual atoms [17] and molecules [18], for example. Hence, the use of tip functionalization in KPFS promises the mapping of charge distributions at ultimate resolution and inside molecules, which is key to the understanding of basic processes in many fields, e.g., organic photovoltaics. Mapping of the quadrupole moment in a molecular switch [4] and of the dipole moment in donor-acceptor molecules [10] has been achieved with CO-terminated AFM tips recently. However, in order to attain charge resolution at the level of individual bonds, the probe has to be brought so close to the sample that other force contributions also become important. In this regime, KPFS maps have shown a contrast inversion [10] which hints that they may not reflect the charge distribution anymore [10].

Here we study trimeric perfluoro-ortho-phenylene mercury $\left(\mathrm{F}_{12} \mathrm{C}_{18} \mathrm{Hg}_{3}\right)$ and its hydrogen-terminated counterpart $\mathrm{H}_{12} \mathrm{C}_{18} \mathrm{Hg}_{3}$, exhibiting bonds of different polarities and intramolecular charge distributions. By analyzing distancedependent KPFS maps of these along with the residuals from fits, we show that KPFS fails to reproduce LCPD in the very-close-distance regime. We identify one physical reason for this limitation of KPFS, which lies in the bias dependence of nonelectrostatic short-range force contributions. To overcome this constraint, we propose a novel FMAFM-based technique to detect the charge distribution on atomic scales. It exploits the dependence of the electric field on tip-sample distance to map out the charge distribution at highest resolution. At intermediate distances, at which KPFS works reliably, it was tested for several different molecules in comparison to KPFS maps. The novel technique allows one to extend the mapping to even closer distances, where KPFS fails. In this regime, we investigate the charge distribution in $\mathrm{F}_{12} \mathrm{C}_{18} \mathrm{Hg}_{3}$ and $\mathrm{H}_{12} \mathrm{C}_{18} \mathrm{Hg}_{3}$, for which contrast changes along individual polar bonds could be resolved.

Experiments were performed using a homebuilt qPlus-based [19] FM-AFM [20] in ultrahigh vacuum $\left(p \approx 5 \times 10^{-11} \mathrm{mbar}\right)$ at low temperatures of $\simeq 5 \mathrm{~K}$ including scanning-tunneling functionality. All investigations of $\mathrm{F}_{12} \mathrm{C}_{18} \mathrm{Hg}_{3}, \mathrm{H}_{12} \mathrm{C}_{18} \mathrm{Hg}_{3}$, and perylene-3,4,9,10tetracarboxylic dianhydride (PTCDA) were performed with the molecules directly adsorbed on $\mathrm{Cu}(111)$, whereas 10-chloro-anthracene-9-carbonitrile $(\mathrm{ClAnCN})$ was studied on a double layer of $\mathrm{NaCl}$ on $\mathrm{Cu}(111)$. The tip was functionalized with a $\mathrm{CO}$ molecule, and bias voltages refer to the sample with respect to the tip. All AFM data were obtained at $0.5 \AA$ oscillation amplitude, and all images and KPFS maps were acquired in constant-height mode. Calculations of $\mathrm{F}_{12} \mathrm{C}_{18} \mathrm{Hg}_{3}$ and $\mathrm{H}_{12} \mathrm{C}_{18} \mathrm{Hg}_{3}$ on $\mathrm{Cu}(111)$ are based on total-energy density-functional theory (DFT) [21] (for details, see Ref. [22]).

To unambiguously relate the experimental data to the polar nature of bonds, we compared $\mathrm{F}_{12} \mathrm{C}_{18} \mathrm{Hg}_{3}$ and $\mathrm{H}_{12} \mathrm{C}_{18} \mathrm{Hg}_{3}$ molecules adsorbed next to each other in the same data set, acquired with the very same tip apex. 

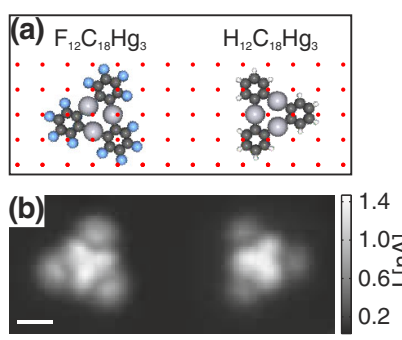

(d)
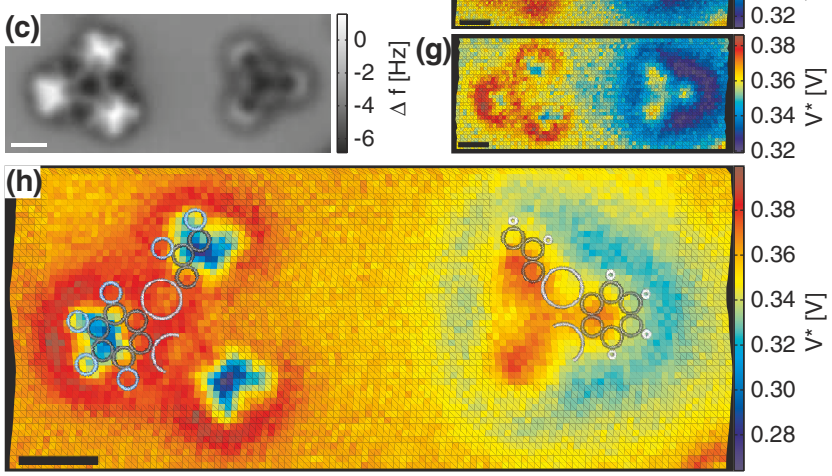

FIG. 1 (color online). KPFS on grids on individual $\mathrm{F}_{12} \mathrm{C}_{18} \mathrm{Hg}_{3}$ and $\mathrm{H}_{12} \mathrm{C}_{18} \mathrm{Hg}_{3}$ molecules. (a) Ball models of $\mathrm{F}_{12} \mathrm{C}_{18} \mathrm{Hg}_{3}$ (lefthand side) and $\mathrm{H}_{12} \mathrm{C}_{18} \mathrm{Hg}_{3}$ (right-hand side) shown in the same orientation as the molecules under investigation. Carbon, mercury, hydrogen, and fluorine atoms are represented in black, gray, white, and blue, respectively. At each point of a dense grid over the molecules (schematically indicated by red dots), individual spectra are acquired. (b) Current image of the two molecules $(z=9.6 \AA \quad[22], \quad V=0.2 \mathrm{~V})$. (c) $\Delta f$ image $(z=9.0 \AA)$. (d) Exemplary KPFS spectrum (blue) of one grid point with parabolic fit (pink) from which $V^{\star}$ is extracted (green). (e)-(h) $V^{\star}$ maps acquired at $z=12.0,10.1,9.8$, and $9.6 \AA$. The experimental data in (h) is partially overlaid with models to indicate the position of individual atoms. All scale bars are $5 \AA$.

Figures 1(a) and 1(c) show the chemical structure and a $\Delta f$ image of both molecules, respectively. To map out the local contact potential difference between the CO-functionalized tip and the sample, KPFS spectra [11] have been acquired on a dense grid of sample points over the molecules [4]. Each KPFS spectrum $\Delta f(V)$ shows a parabolic shape [see Fig. 1(d)] and from a fit to a parabola, the voltage $V^{\star}$, at which $\Delta f(V)$ has its maximum, is extracted. Usually, $V^{\star}$ is assumed to equal the voltage of compensated LCPD, such that $V_{\mathrm{LCPD}}=V^{\star}$ [11]. Figures 1(e)-1(f) show the maps of $V^{\star}$ values with decreasing tip-sample distance. For relatively large distances of $12.0 \AA$, these $V^{\star}$ maps show a contrast that is expected from an interface dipole formation [15]. For the remainder, it is important that any charge at the surface of the metal substrate will be screened efficiently (the image-charge effect) leading to a dipole rather than an isolated point charge [30]. Because of this and the superposition principle, any distribution of charges at a metal surface can equally be described by a set of dipoles, each perpendicular to the surface; see the inset in Fig. 2(a). Each of these dipoles will change the LCPD. This highlights the direct link between charges inside the adsorbate, the resulting surface dipoles, and LCPD. Upon approaching the surface slightly, Fig. 1(f), the $V^{\star}$ contrast increases and shows higher lateral resolution. The largest shifts of the $V^{\star}$ signal are observed at the circumference of the molecule. In agreement with this observation, our DFT simulations reveal that the interface dipoles at the two molecules of $-1.16 D$ and $+3.87 D$, respectively, are mainly due to vertical relaxations of the polar peripheral bonds of opposite polarity $\left(\mathrm{C}^{\delta+}-\mathrm{F}^{\delta-} \mathrm{vsC}^{\delta-}-\mathrm{H}^{\delta+}\right)$; see Fig. S5 in Ref. [22]. At the closest distance in the experiment, Fig. 1(h), mercury atoms within $\mathrm{F}_{12} \mathrm{C}_{18} \mathrm{Hg}_{3}$ become apparent. In addition, the contrast inverses at the outer part of the phenylene groups, where it shows sharp red to blue transitions. Although this intramolecular contrast is remarkable, such a contrast inversion [10] is incompatible with the simple interpretation in terms of LCPD [22].

One possible contribution to the KPFS signal at close distances is the so-called phantom force [32] resulting from the current. However, a strong influence of phantom force can be excluded here, because (i) the tunneling current image [Fig. 1(b)] bears no resemblance to the features of the contrast inversion, and (ii) the tunneling current is similar for both molecules, whereas $V^{\star}$ is strikingly different.

Instead, it has been speculated that the contrast inversion stems from the probe penetrating the molecule's electron shell so that the unscreened core charges contribute to the KPFS signal [10]. Electrostatic force contributions give rise to the parabolic dependence of $\Delta f(V)[11,15]$. From the above reasoning, no deviation from a parabolic shape is expected. Therefore, we analyzed the residuals of the parabolic fit to the KPFS spectra for different distances, as is shown in Fig. 2(b). Whereas, at large distances, the residuum is a random noise signal-it shows structure not compatible with noise at close distances. This systematic deviation from a parabolic shape points toward a contribution to the signal involving not only electrostatics.

The large electric fields in the junction on the order of $10^{9} \mathrm{~V} / \mathrm{m}$ that occur upon acquisition of a KPFS parabola will lead to a relaxation of the positions of atoms that carry net charge (ionic polarization), and a Stark effect of the electron shell (electronic polarization). Both effects will affect the overlap of the electron shells of tip and sample, contributing to the Pauli repulsion and hence to $\Delta f$ in the close-distance regime [33]. As the Pauli repulsion is very short range in nature, even tiny relaxations lead to an appreciable signal. The above considerations provide a good reason why KPFS fails to reproduce LCPD in this regime. Upon sweeping the bias voltage, the sample and the tip will undergo relaxations affecting the Pauli repulsion and hence the frequency shift. Since this involves nonelectrostatic interactions, a deviation from a parabolic shape of $\Delta f(V)$ can be expected. Even if this deviation is not immediately apparent from looking at the data, the fit of $V^{\star}$ might be heavily affected. To analyze this, we 

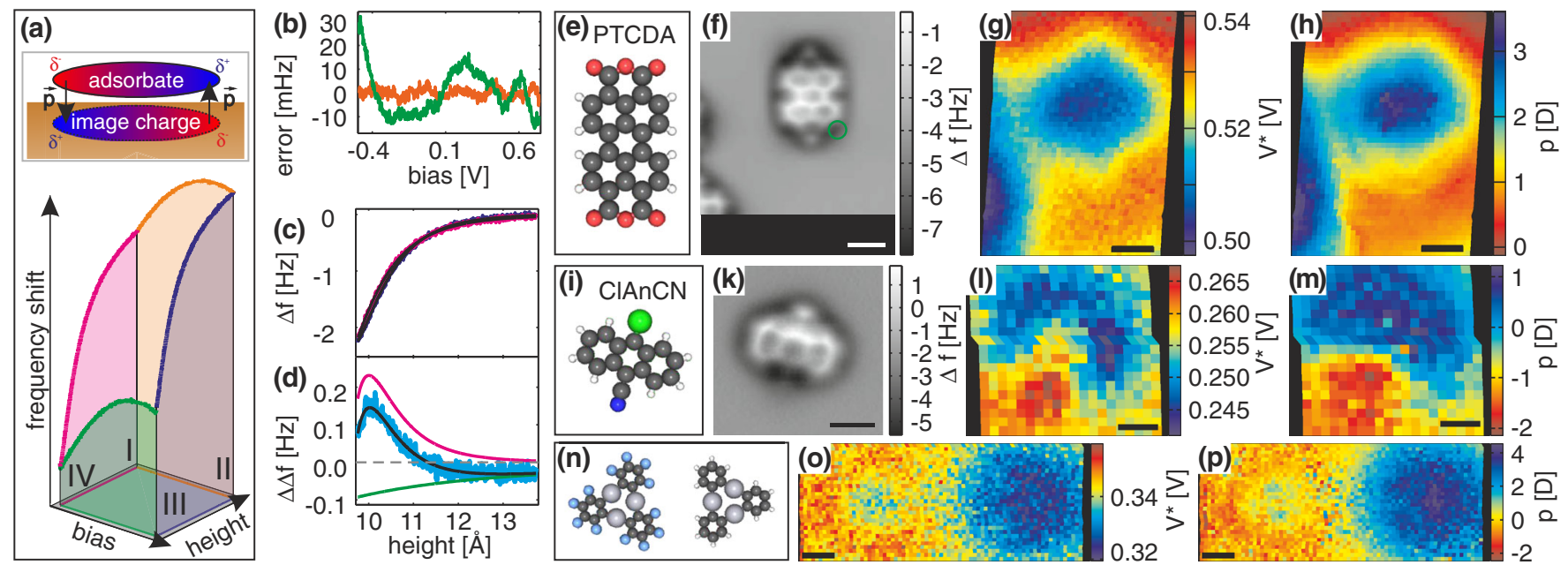

FIG. 2 (color online). Deducing intramolecular charge distribution from $z$ ramps. (a) Schematic illustration of the data acquisition procedure: For each lateral grid position, two KPFS parabolas at different distances (I $\rightarrow$ II and III $\rightarrow$ IV) and two $\Delta f(z)$ curves at different voltages (II $\rightarrow$ III and IV $\rightarrow$ I) are recorded. (b) Residuals of the parabolic fit to KPFS data show just random noise for a large tip-sample spacing (orange) but systematic deviations for a short one (green). (Data points smoothed over $75 \mathrm{mV}$.) (c) Background subtracted $\Delta f\left(z, V_{i}\right)$ curves along with their Lennard-Jones fits. (d) The difference spectrum $\Delta \Delta f(z, \Delta V)$ (blue) is fitted by contributions from electrostatics (green) and vertical relaxation (pink), the sum of which is shown in black (the dashed gray line indicates zero). The green marker in (f) indicates the lateral position of the spectra shown in (a)-(d). (e)-(p) Comparison of dipoledistribution maps extracted from $\Delta f\left(z, V_{i}\right)$ spectra $[(\mathrm{h}),(\mathrm{m}),(\mathrm{p})]$ and conventional KPFS maps $[(\mathrm{g}),(\mathrm{l})$, (o)] at relatively large distances for PTCDA [(e)-(h)], ClAnCN [(i)-(m)], and $\mathrm{F}_{12} \mathrm{C}_{18} \mathrm{Hg}_{3}$ and $\mathrm{H}_{12} \mathrm{C}_{18} \mathrm{Hg}_{3}$ [(n)-(p)] [31].

evaluated the goodness of the parabolic fit to the experimental $\Delta f(V)$ spectrum for each pixel of Fig. 1(h), revealing that the contrast inversion is spatially correlated with systematic deviations from parabolas; see Fig. S1 in Ref. [22].

Whereas the above considerations hint toward a fundamental limitation of KPFS to reproduce LCPD at very close distances, they offer a route to overcome the limitations of KPFS in charge detection at very close distances, as follows. The basic idea of the novel scheme is to better disentangle electrostatic from nonelectrostatic contributions of $\Delta f$ from their different distance dependence [34]. In addition to KPFS spectra $\Delta f(V)$ (at a given tip height), we acquired $\Delta f(z)$ data for two distinctly different bias voltages $V_{i}$; see the schematic in Fig. 2(a). Both of these $\Delta f(z)$ curves are expected to display the distance dependence of the forces (or-more precisely-the tipsample stiffness $\partial F_{z} / \partial z$ ) between tip and sample molecule. The interactions other than electrostatic in origin can be modeled by a Lennard-Jones potential; we therefore refer to the part of the $\Delta f(z)$ signal resulting from these contributions as $\Delta f_{\mathrm{LJ}}(z)$. A vertical relaxation $\delta z$ resulting from the drastic change of bias voltage will-in good approximation [22] - simply shift the two $\Delta f(z)$ curves with respect to the vertical position $z$, so that $\Delta f_{\mathrm{LJ}}\left(z, V_{2}\right) \simeq$ $\Delta f_{\mathrm{LJ}}\left(z+\delta z, V_{1}\right)$. As these relaxations are expected to be small, a Taylor expansion yields that the difference $\Delta f_{\mathrm{LJ}}\left(z, V_{2}\right)-\Delta f_{\mathrm{LJ}}\left(z, V_{1}\right) \simeq \Delta f_{\mathrm{LJ}}\left(z+\delta z, V_{1}\right)-\Delta f_{\mathrm{LJ}}\left(z, V_{1}\right) \simeq$ $\delta z \cdot \Delta f_{\mathrm{LJ}}^{\prime}\left(z, V_{1}\right)$ equals the product of $\delta z$ times the derivative $\Delta f_{\mathrm{LJ}}^{\prime}\left(z, V_{1}\right)=\partial \Delta f_{\mathrm{LJ}}\left(z, V_{1}\right) / \partial z$. Hence, the extraction of the difference signal may directly provide information about the polarization of molecules on surfaces.

Moreover, the two $\Delta f(z)$ spectra will also contain several electrostatic contributions which can be classified $[35,36]$ in the ones that stem from the overall electrostatic tip-substrate interaction and those from local charges in the junction. The former can be eliminated by subtracting the $\Delta f(z)$ signal acquired away from the molecule on the bare substrate from each of the spectra at the given voltage. Henceforth, $\Delta f(z)$ refers to the background subtracted signal. Note that this background subtraction will not affect the considerations discussed in the previous paragraph. What remains are the contributions from local charges inside the junction. We make use of the fact that in the presence of the metal, they can be described by a set of vertical dipoles (see above). Each of these dipoles interacts with a local electric field in the junction. Even though the electric field is enhanced locally right beneath the tip [see Fig. S7 in Ref. [22]], we approximate it for simplicity as $\left(V_{\text {bias }}-\mathrm{LCPD}\right) / z$, where $z$ is the tipsubstrate distance [37].

As the surface dipole $p$ and the electric field right beneath the functionalized tip are both oriented perpendicular to the surface, their interaction potential equals $p\left(V_{\text {bias }}-\mathrm{LCPD}\right) / z$. Differentiating this expression twice yields a corresponding $\Delta f(z)$ contribution, which is proportional to $p\left(V_{\text {bias }}-\right.$ LCPD $) / z^{3}$. Hence, the difference spectrum $\Delta \Delta f(z, \Delta V)=\Delta f\left(z, V_{2}\right)-\Delta f\left(z, V_{1}\right)$ is expected to show two contributions: one being proportional to $\delta z \Delta f^{\prime}\left(z, V_{1}\right)$ [due to vertical relaxation $\delta z$, the pink curve in Fig. 2(d)] and a second one proportional to $p \Delta V / z^{3}$ (the 
green curve) indicating the presence of surface dipoles $p$ in the junction [38]. Note that the functional dependences on $z$ of these two contributions are qualitatively different from each other and therefore allow one to discriminate between the two in a fitting procedure [39]. One has to be aware that the above proposed novel method and fitting procedure involve quite some simplifications and approximations [22].

Although we believe that extracting the bias-dependent vertical relaxation $\delta z$ from these fits may turn out to be useful in the future, in the current experiment we attribute any such relaxations mainly to the $\mathrm{CO}$ at the tip [33] rather than to the molecule on the surface. We therefore disregard the relaxation $\delta z$ and concentrate on the dipole maps only. Figure 2 displays the experiments following the scheme described above. For each point on a dense grid, we recorded the frequency shift as a function of the tip-sample spacing at two different bias voltages, as is exemplarily displayed for one individual grid point in a 3D representation in Fig. 2(a). The two spectra $\Delta f\left(z, V_{1}\right)$ and $\Delta f\left(z, V_{2}\right)$ are depicted in Fig. 2(c). Despite their similarity, the difference signal $\Delta \Delta f(z, \Delta V)$ displayed in Fig. 2(d) still shows distinct features. A comparison to the derivative $\Delta f^{\prime}\left(z, V_{1}\right)$ (pink) shows striking agreement in the overall shape, which we view as a confirmation of the relaxation effect, as described above. The offset between the experimental data and $\Delta f^{\prime}\left(z, V_{1}\right)$ indicates clearly that another contribution is missing. An additional contribution proportional to $-\Delta V / z^{3}$ (green) results in a sum (black) that excellently fits the data. From this fitting procedure, the dipole $p$ is extracted for each grid point, rendering maps, which are henceforth referred to as dipole maps. To test the novel method, we compared such dipole maps to maps of $V^{\star}=V_{\mathrm{LCPD}}$ from KPFS at distances at which KPFS works reliably. The resulting maps are displayed in Figs. 2(e)-2(p) for PTCDA on copper, $\mathrm{ClAnCN}$ on the $\mathrm{NaCl}$ bilayer on copper, and for $\mathrm{F}_{12} \mathrm{C}_{18} \mathrm{Hg}_{3}$ and $\mathrm{H}_{12} \mathrm{C}_{18} \mathrm{Hg}_{3}$ on copper, along with a model of their chemical structure and a $\Delta f$ image. In the case of $\mathrm{ClAnCN}$, the $\mathrm{NaCl}$ bilayer was introduced to benchmark the method also for a distinctly different electronic coupling and current regime. In all cases the dipole maps [Figs. 2(h), 2(m), and 2(p)] closely resemble the $V^{\star}$ maps [cf. Figs. 2(g), 2(1), and 2(o)]. In turn, in this regime $V^{\star}$ maps are assumed to reflect the local charge distribution $[4,10,15]$.

Lateral resolution and contrast increase drastically when the tip-sample spacing is reduced. A corresponding dipole map for $\mathrm{F}_{12} \mathrm{C}_{18} \mathrm{Hg}_{3}$ and $\mathrm{H}_{12} \mathrm{C}_{18} \mathrm{Hg}_{3}$ on copper is displayed in Fig. 3, along with a $\Delta f$ image and the DFT calculated charge distribution, for comparison. Note that the dipole maps are expected to be a convolution of the local dipoles with the lateral profile of the electric field beneath the tip [40], smearing out the apparent dipole distribution. Remarkably, this map still shows pronounced intramolecular contrast without suffering from similar artifacts as the KPFS-derived map shown in Fig. 1(h). Interestingly, at the positions of the
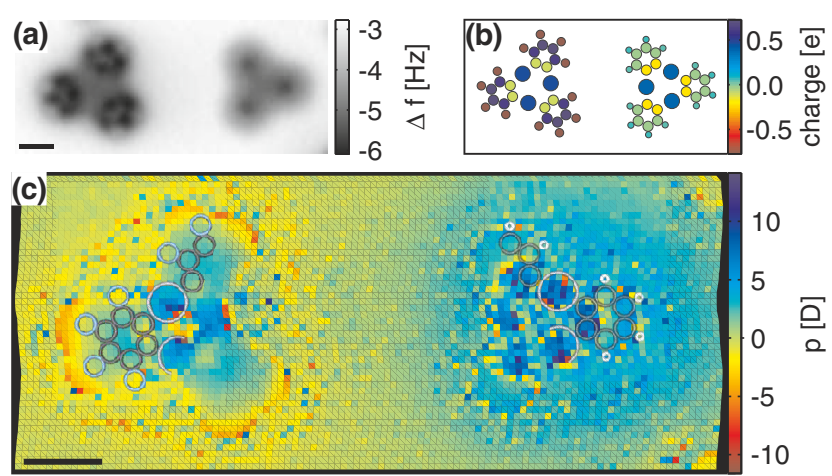

FIG. 3 (color online). Highly resolved dipole-distribution map. (a) $\Delta f$ image recorded at $z=9.6 \AA$. (b) Calculated charge distribution deduced from Bader analysis. (For details, see Table S1 in Ref. [22].) (c) Dipole-distribution map extracted from $\Delta f\left(z, V_{i}\right)$ spectra for $\mathrm{F}_{12} \mathrm{C}_{18} \mathrm{Hg}_{3}$ and $\mathrm{H}_{12} \mathrm{C}_{18} \mathrm{Hg}_{3}$ $\left(9.6 \AA \leq z \leq 10.1 \AA ; V_{i}=-0.2\right.$ and $\left.0.5 \mathrm{~V}\right)$.

mercury atoms, the contrast in this map is much stronger than in the $\Delta f$ images [cf. Figs. 1(c) and 3(a)].

The dipole map shows a contrast that is in agreement with the calculated charge distribution [see Fig. 3(b)] and the electrostatic potential map (see Ref. [22]). The contrast observed at the circumference of the molecules fits to the overall surface dipole as discussed above. Directly along the C-F bonds a strong contrast change from green to red indicates the electrons being pulled toward the fluorine $\left(\mathrm{C}^{\delta+}-\mathrm{F}^{\delta-}\right)$. The $\mathrm{C}-\mathrm{H}$ bonds show much less of a polar nature, with a tendency toward $\mathrm{C}^{\delta-}-\mathrm{H}^{\delta+}$ in accordance with the Bader analysis [see Fig. 3(b)]. The map also reveals that the mercury atoms on both molecules apparently carry net positive charge, in good agreement with DFT. The signal on benzene rings shows relatively weak contrast with respect to $\mathrm{Hg}$ atoms, in fair agreement with the calculations.

It needs to be emphasized that the dipole maps-even though provided in units of Debye - may not be taken as being quantitative. Experimental uncertainties in determining the absolute tip-sample distance, averaging effects, the bending of the $\mathrm{CO}$ molecule at the tip apex, the approximations made in the fitting procedure, and tip-induced surface dipoles may influence the quantitative interpretation. Nonetheless, we are convinced that this novel technique presents a route to better disentangle electrostatic from nonelectrostatic contributions to overcome the limitations of KPFS at closest distances and highest resolution. It may even be a first step toward a quantitative determination of surface dipoles.

In conclusion, the experiments presented here provide insight as to why KPFS systematically fails to reproduce the LPCD at very close tip-sample distances. We introduce a new method that allows for resolving intramolecular charge distributions with unprecedented resolution, showing contrast at the atomic scale. 
We thank Andreas Pöllmann, François P. Gabbaï, John McManus, Leo Gross, Niko Pavliček, Norio Okabayashi, Pingo Mutombo, and Prokop Hapala for the fruitful discussions and the help. We greatly appreciate the support from the National Grid Infrastructure MetaCentrum (Project No. LM2010005) for our calculations. We are grateful for funding by the DFG and the GAČR (through Research Training Group 1570 and Grand No. Sche 384/ 26-2 and the German-Czech bilateral Project Nos. RE2669/ $4 ; 14-16963 \mathrm{~J}$ ) and the Volkswagen foundation (through its "Lichtenberg" program).

*Corresponding author. Jascha.Repp@ur.de

[1] L. Gross, F. Mohn, N. Moll, P. Liljeroth, and G. Meyer, Science 325, 1110 (2009).

[2] L. Gross, F. Mohn, N. Moll, G. Meyer, R. Ebel, W. M. Abdel-Mageed, and M. Jaspars, Nat. Chem. 2, 821 (2010).

[3] Z. Sun, M. P. Boneschanscher, I. Swart, D. Vanmaekelbergh, and P. Liljeroth, Phys. Rev. Lett. 106, 046104 (2011).

[4] F. Mohn, L. Gross, N. Moll, and G. Meyer, Nat. Nanotechnol. 7, 227 (2012).

[5] N. Pavliček, B. Fleury, M. Neu, J. Niedenführ, C. HerranzLancho, M. Ruben, and J. Repp, Phys. Rev. Lett. 108, 086101 (2012).

[6] J. Welker and F. J. Giessibl, Science 336, 444 (2012).

[7] F. Albrecht, M. Neu, C. Quest, I. Swart, and J. Repp, J. Am. Chem. Soc. 135, 9200 (2013).

[8] D. G. de Oteyza, P. Gorman, Y.-C. Chen, S. Wickenburg, A. Riss, D. J. Mowbray, G. Etkin, Z. Pedramrazi, H.-Z. Tsai, A. Rubio, M. F. Crommie, and F. R. Fischer, Science 340, 1434 (2013).

[9] J. van der Lit, M. P. Boneschanscher, D. Vanmaekelbergh, M. Ijäs, A. Uppstu, M. Ervasti, A. Harju, P. Liljeroth, and I. Swart, Nat. Commun. 4, 2023 (2013).

[10] B. Schuler, S.-X. Liu, Y. Geng, S. Decurtins, G. Meyer, and L. Gross, Nano Lett. 14, 3342 (2014).

[11] M. Nonnenmacher, M. P. O'Boyle, and H. K. Wickramasinghe, Appl. Phys. Lett. 58, 2921 (1991).

[12] R. Bennewitz, M. Bammerlin, M. Guggisberg, C. Loppacher, A. Baratoff, E. Meyer, and H.-J. Güntherodt, Surf. Interface Anal. 27, 462 (1999).

[13] S. A. Burke, J. M. LeDue, Y. Miyahara, J. M. Topple, S. Fostner, and P. Grütter, Nanotechnology 20, 264012 (2009).

[14] S. Sadewasser, P. Jelinek, C.-K. Fang, O. Custance, Y. Yamada, Y. Sugimoto, M. Abe, and S. Morita, Phys. Rev. Lett. 103, 266103 (2009).

[15] J. L. Neff and P. Rahe, Phys. Rev. B 91, 085424 (2015).

[16] R. Stomp, Y. Miyahara, S. Schaer, Q. Sun, H. Guo, P. Grutter, S. Studenikin, P. Poole, and A. Sachrajda, Phys. Rev. Lett. 94, 056802 (2005).

[17] L. Gross, F. Mohn, P. Liljeroth, J. Repp, F. J. Giessibl, and G. Meyer, Science 324, 1428 (2009).

[18] T. Leoni, O. Guillermet, H. Walch, V. Langlais, A. Scheuermann, J. Bonvoisin, and S. Gauthier, Phys. Rev. Lett. 106, 216103 (2011).
[19] F. J. Giessibl, Appl. Phys. Lett. 76, 1470 (2000).

[20] T. R. Albrecht, P. Grütter, D. Horne, and D. Rugar, J. Appl. Phys. 69, 668 (1991).

[21] G. Kresse and J. Furthmüller, Phys. Rev. B 54, 11169 (1996).

[22] See Supplemental Material at http://link.aps.org/ supplemental/10.1103/PhysRevLett.115.076101, which includes Refs. [23-29], for details on methods and approximations used and for additional results of both measurement and calculations.

[23] U. Dürig, H. R. Steinauer, and N. Blanc, J. Appl. Phys. 82, 3641 (1997).

[24] P. Sartori and A. Golloch, Chem. Ber. 101, 2004 (1968).

[25] G. Wittig and F. Bickelhaupt, Chem. Ber. 91, 883 (1958).

[26] T. J. Taylor, C. N. Burress, and F. P. Gabbaï, Organometallics 26, 5252 (2007).

[27] D. Vanderbilt, Phys. Rev. B 41, 7892 (1990).

[28] J. P. Perdew, J. A. Chevary, S. H. Vosko, K. A. Jackson, M. R. Pederson, D. J. Singh, and C. Fiolhais, Phys. Rev. B 48, 4978 (1993).

[29] G. Henkelman, A. Arnaldsson, and H. Jónsson, Comput. Mater. Sci. 36, 354 (2006).

[30] J. D. Jackson, Classical Electrodynamics (Wiley, New York, 2001).

[31] Tip heights for PTCDA: $\Delta f$ image $9.0 \AA, V^{\star}$ map $12.6 \AA$, charge map $z$ sweep between 12.6 and $9.6 \AA$ at -0.4 and $0.75 \mathrm{~V}$ bias. Tip heights for ClAnCN: $\Delta f$ image at $15.4 \AA$, $V^{\star}$ map at $18.2 \AA$, charge map $z$ sweep between 18.2 and $16.2 \AA$ at -0.5 and $0.5 \mathrm{~V}$ bias. Tip heights for $\mathrm{F}_{12} \mathrm{C}_{18} \mathrm{Hg}_{3}$ and $\mathrm{H}_{12} \mathrm{C}_{18} \mathrm{Hg}_{3}: V^{\star}$ map at $11.0 \AA$, charge map $z$ sweep between 11.5 and $9.9 \AA$ at -0.2 and $0.35 \mathrm{~V}$ bias.

[32] A. J. Weymouth, T. Wutscher, J. Welker, T. Hofmann, and F. J. Giessibl, Phys. Rev. Lett. 106, 226801 (2011).

[33] N. Moll, L. Gross, F. Mohn, A. Curioni, and G. Meyer, New J. Phys. 14, 083023 (2012).

[34] M. A. Lantz, H. J. Hug, R. Hoffmann, P. J. A. van Schendel, P. Kappenberger, S. Martin, A. Baratoff, and H.-J. Güntherodt, Science 291, 2580 (2001).

[35] L. Gross, B. Schuler, F. Mohn, N. Moll, N. Pavliček, W. Steurer, I. Scivetti, K. Kotsis, M. Persson, and G. Meyer, Phys. Rev. B 90, 155455 (2014).

[36] A. Sadeghi, A. Baratoff, S. A. Ghasemi, S. Goedecker, T. Glatzel, S. Kawai, and E. Meyer, Phys. Rev. B 86, 075407 (2012).

[37] Note that the dipole under consideration is in direct proximity to the sample, where this approximation is justified. For an estimate of the vertical tip-sample distance, see Ref. [22].

[38] For the conversion factor to extract the dipole in physically meaningful units, see Ref. [22].

[39] Changing the exponents of the Lennard-Jones fit from a (12-6) potential to a (12-4) potential did not affect the fit result for the dipole maps qualitatively.

[40] D. Z. Gao, J. Grenz, M. B. Watkins, F. Federici Canova, A. Schwarz, R. Wiesendanger, and A. L. Shluger, ACS Nano 8, 5339 (2014). 